\title{
Factors associated with differential community prevalence of hepatitis $E$ in two sub-counties in Kitgum District in Northern Uganda: A comparative cross-sectional study
}

Judith Aloyo ( $\square$ aloyo2011@gmail.com )

Rhites-N, Acholi

Juliet Kiguli

Makerere University School of Public Health, Kampala, Uganda

Christopher Orach Garimoi

Makerere University, School of Public Health, Department of Community and Behavioral Sciences,

Kampala, Uganda

Eric Nzirakaindi Ikoona

ICAP at Columbia University, Sierra Leone

David Lagoro Kitara

Gulu University, Faculty of Medicine, Department of Surgery https://orcid.org/0000-0001-7282-5026

\section{Research Article}

Keywords: Hepatitis E, prevalence, personal hygiene, community awareness and practices, Kitgum

Posted Date: December 20th, 2021

DOI: https://doi.org/10.21203/rs.3.rs-1182084/v1

License: (c) (i) This work is licensed under a Creative Commons Attribution 4.0 International License.

Read Full License 


\section{Abstract}

Background: Hepatitis $E$ is one of the leading causes of acute viral hepatitis worldwide. During 2009, an epidemic of hepatitis E resulted in 10,437 infections and 167 deaths in Kitgum district.

Objective: To investigate factors associated with the differential community prevalence of hepatitis $E$ in two sub-counties in Kitgum District.

Methods: We conducted a community survey during the $4^{\text {th }}-31^{\text {st }}$ of May 2012 in two Sub Counties in Kitgum District in Northern Uganda. A total of 474 heads of household were recruited using a probability proportional to size through multistage and random sampling methods. Two hundred thirty-four (49.26\%) heads of household were from Mucwini, and 241 (50.74\%) were from Kitgum Matidi Sub Counties. The questionnaire had an internal validity of Cronbach's $a=0.85$. The study was approved by a local IRB. STATA version 10.0 was used for data analysis, and a p-value less than 0.05 was considered significant.

Results: The prevalence of hepatitis E was significantly higher in Mucwini Subcountry 97 (41.99\%) than in Kitgum Matidi $63(26.47 \%) ; \chi^{2}=12.6 ; p=0.000$. Factors associated with differential prevalence were hand washing after latrine use with Adjusted Odds Ratio (AoR) $=0.23,95 \% \mathrm{Cl}: 0.110-0.646 ; p=0.003$; frequency of communal hand washing $A o R=0.53,95 \% \mathrm{Cl}: 0.330-0.860 ; p=0.01$; patients' handling by health workers $\mathrm{AoR}=1.91,95 \% \mathrm{Cl}: 1.410-2.610 ; \mathrm{p}<0.001$; frequency of village health meetings held AoR $=0.69,95 \% \mathrm{Cl}: 0.56-0.85 ; p<0.001$ and awareness of the cause of Hepatitis $E$ AoR $=1.42,95 \% \mathrm{Cl}: 0.710-$ $1.880 ; p=0.015$.

Conclusions: Factors associated with the differential community prevalence of hepatitis $E$ in the two communities were poor personal hygiene, poor community practices and a low level of community awareness about the virus. District and health authorities should put in place measures to improve personal and household hygiene and strengthen community health education on hepatitis $\mathrm{E}$.

\section{Introduction}

Hepatitis E virus (HEV) is one of the leading causes of acute viral hepatitis in the world and is prevalent primarily in Africa, Central Asia and Mexico. ${ }^{1}$ Hepatitis E virus is a nonenveloped RNA virus belonging to the genius herpesvirus family that was first recognized in early $1980 .^{2}$ The highest prevalence of infection is usually found in regions where there are low standards of sanitation that promote the transmission of the virus. ${ }^{1}$ The virus is largely spread via the orofecal route and presents as an acute, self-limiting gastrointestinal illness that affects both sexes with high age-specific attack rates among persons aged between $15-45$ years. ${ }^{3}$ The case fatality rate is generally as high as $20 \%$ in women during their third trimester of pregnancy. ${ }^{4}$

The main symptoms in the early phases of the disease include anorexia, fatigue, myalgia, weight loss, distaste of food and nausea, which is usually followed by jaundice after one to two weeks. ${ }^{1,3,5}$ Headache, 
arthralgia, vomiting and upper quadrant tenderness are manifestations that occur later as the disease progresses. ${ }^{1,3,5}$ During the icteric phase, patients have dark brown urine and clay-colored stools, and diarrhea is a common presentation specifically in children. ${ }^{1,3}$

A rare but frequently fatal form of acute hepatitis $E$ in which a patient's condition rapidly deteriorates with hepatic encephalopathy, necrosis of the hepatic parenchyma, coagulopathy, renal failure, and coma with mortality rates ranging between $0.5 \%$ and $4.0 \%$ has been reported in the overall population. ${ }^{1,6}$

Additionally, complications, including premature deliveries with high infant mortality rates, abortions, and maternal deaths, have been reported. ${ }^{1}$ However, the reason for the high mortality is not yet clear, as researchers have attributed it to toxemia with hypertension, proteinuria, edema, and kidney lesions. ${ }^{1}$

By directly or indirectly affecting the kidneys, hepatitis E might precipitate eclampsia and may be the cause of the increased mortality rates in pregnant women. ${ }^{1}$ To date, there are no identified chronic sequelae of hepatitis $\mathrm{E}$ infection. ${ }^{1}$

Fascinatingly, very little is known about the available treatment capable of altering the course of acute hepatitis resulting from hepatitis $E$ infection. ${ }^{1,6}$ Measures to control and prevent transmission of hepatitis E mainly depend on the provision of clean water and proper sanitation. ${ }^{1,6}$ Additional strategies for prevention and control include avoiding drinking water of unknown purity and eating uncooked fish, fruits, and vegetables. ${ }^{1}$ Secondary prevention requires early identification of cases using epidemiological surveillance and interventions on patients in the early stages of the disease, which include early diagnosis when the presence of IgM anti-HEV can be detected in the laboratory. ${ }^{1}$ Tertiary prevention requires actions that prevent further deterioration of the patient and reduce complications on the liver by interventions such as liver transplant. ${ }^{1}$

To date, there are no known curative measures or commercially available vaccines that are viable and effectively used against hepatitis E. ${ }^{1,6}$ However, in 2011, the first vaccine to prevent hepatitis E infection was listed in China. ${ }^{7}$ Although the vaccine is not yet available globally, it could potentially become available in several countries in a few months to years to come. ${ }^{7}$

Additionally, the first retrospectively documented outbreak of hepatitis E occurred in India during 19551956. ${ }^{8}$ Since then, large outbreaks have been reported in Asia and Africa in contrast to sporadic cases seen in industrialized countries. ${ }^{9-10,11}$ The virus is classified into 4 genotypes and presents as two different conditions: large epidemics and sporadic cases. ${ }^{12}$ To date, genotypes 1 and 2 have been found only in humans, whereas genotypes 3 and 4 have been found in animals. ${ }^{13}$

The 2007 outbreak of hepatitis E, which occurred in rural communities in internally displaced persons (IDP) camps of Madi Opei Sub County, Lamwo District in Northern Uganda, was caused by HEV (genotype 1). ${ }^{14}$ That epidemic affected a number of children less than 2 years (6.9\%) but also more 
women $(28 \%)$ than men $(22 \%) .{ }^{14}$ In that outbreak, the highest number of symptomatic cases of hepatitis E were reported in pregnant women (80.7\%). ${ }^{14,15} \mathrm{It}$ was stated that by 2009 , an estimated 10,437 persons were reported to have acquired hepatitis E infection, leading to 167 deaths with a case fatality rate of $1.59 \% .{ }^{16}$ The factors associated with the transmission of hepatitis $E$ in that outbreak were poor hygiene coupled with poor water storage practices and person-to-person transmission. ${ }^{17}$

A review of the Kitgum District Health Management Information System (HMIS) of 2010 showed a persistently higher number of hepatitis E cases reported in Mucwini Sub County, where between 3-6 cases were registered weekly compared to 0-3 cases in Kitgum Matidi Sub County. ${ }^{18}$

The objective of this study was to investigate factors associated with the differential community prevalence of hepatitis $E$ in two sub-counties in Kitgum District to inform strategies for hepatitis $E$ prevention and control in the district.

\section{Materials And Methods}

\section{Study design}

This was a comparative cross-sectional study design conducted between the 4th and 31st of May 2012.

\section{Study Settings}

This study was conducted in Mucwini and Kitgum Matidi Sub Counties in Kitgum District in Northern Uganda. Kitgum district is located approximately 452 kilometers by road, north of Kampala, the capital city of Uganda and is bordered to the north by Lamwo and South Sudan, Kaabong and Kotido districts to the East, Agago and Pader districts to the South, and Gulu district to the West (Figure 1). Kitgum District has one county-Chua with 10 sub counties, 44 parishes and 437 villages. Mucwini Sub County, where the study was conducted, had a population of 23,423 inhabitants with 9 parishes and 65 villages, while Kitgum Matidi had a population of 20,356 inhabitants with 4 parishes and 47 villages.

The district has eight health Centre III (HCIII), one health Centre 4 (HC1V) at the health subdistrict level (Namukora HC IV) and one government hospital. Kitgum Government Hospital is a general hospital and a public facility with 360 beds, and another, a private but not-for-profit hospital (PNP), St. Joseph's Hospital which is a 250-bed facility. The district's main economic activity is subsistence farming, which is dependent mainly on the two seasonal climatic variations in the region. The two sub-counties were purposefully chosen for this study because they differed in the number of registered hepatitis $\mathrm{E}$ cases reported monthly to the Kitgum district surveillance office.

\section{Study population}

The study population comprised heads of household in Mucwini and Kitgum Matidi Sub Counties in Kitgum District. 


\section{Selection criteria}

\section{Inclusion criteria}

Male or female heads of household aged 18 years and above, informed consent to participate, and residing in the two sub-counties from the onset of the hepatitis $E$ epidemic.

\section{Exclusion criteria}

Heads of households who were absent from home, drunk or acutely ill to participate in this study.

\section{Sample size estimation}

We calculated the sample size for this study using a formula for sample size estimation when evaluating differences between two proportions. This sample size was calculated using a formula by Altman $\mathrm{G}$ Douglas (1995); Bland, 2000). ${ }^{19,20}$

In each group

\section{$n=\left(p_{0} q_{0}+p_{1} q_{1}\right)+(f \alpha$, power $)$ $\left(p_{1}-p_{0}\right)^{2}$}

Where:

$P_{1}=$ prevalence of hepatitis $E$ in Mucwini Sub County taken at 61\% (Kitgum district HMIS, 2009).

$\mathrm{P}_{0}=$ prevalence of hepatitis $\mathrm{E}$ in Kitgum Matidi Sub County taken at 46\% (Kitgum district HMIS, 2009).

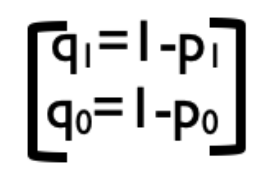

$f(a$, power $)=a$ function of power and level of significance or type 1 error which is taken at 7.9 for the power of $80 \%$, a $p_{1}$ of $61 \%$ and a $p_{0}$ of $46 \%$, the sample size in each arm at $5 \%$ level of significance.

Substituting in the formula, the number of respondents in each Sub County is .

$(0.46 \times 0.54)+(0.61 \times 0.39) 7.9=170.75$ $(0.6 \mathrm{I}-0.46)^{2}$

respondents per Sub County

Using the design effect default value of 1.3 (to compensate for the use of cluster sampling), according to the Uganda Demographic Health Survey (UDHS) of 2006, the total number of participants from each Sub County was estimated as $n \times D$, which was $1.3 \times 170.75=221.98$. Thus, the total sample size of the study population was estimated at 443.96 participants. 
To cater to nonresponses and recording errors, $5 \%$ was added $(n+5 \%)$

Thus, $=443.96 / 0.95=467.3$, which was rounded off to 480 participants.

The number of households visited per village was $480 / 30=16$ households per village.

Therefore, in each Sub County, 15 villages were sampled, and 240 participants were interviewed.

\section{Sampling and field procedures}

The two sub counties of Mucwini and Kitgum Matidi were purposely selected because they differed in the number of hepatitis E cases reported in the health management information system (HMIS) of Kitum District. Mucwini Sub County had 9 parishes and a total of 65 villages, while Kitgum Matidi Sub County had 4 parishes and 47 villages.

\section{Selection of villages in the two Sub Counties}

Villages (clusters) and households (study unit) were selected by means of two-stage and cluster sampling methods using the WHO EPI method (WHO 1991). ${ }^{21}$ Villages were selected using a probability proportional to size (PPS) method. This sampling approach was adopted because sampling units (villages) varied considerably in size to ensure that larger and smaller villages had the same probability of entering the sampled population. In addition, this method facilitated our planning and allocation of staff for the field work, as we prepared for interviews with respondents in each of the selected units. A total of 30 clusters (villages) were used as the final groupings for the sampling in the two sub counties.

To achieve the selection of study clusters in Mucwini Sub County, a list of villages and population sizes were obtained from Mucwini Sub County headquarters and fixed in columns A and B, respectively. The cumulative population was run in column $\mathrm{C}$, giving the total population of Mucwini as the number in column C. The total population of Mucwini was divided by 15 (the number of villages that were visited), and the resultant figure was the sampling interval ( $\mathrm{SI}$ ). A number between one and the sampling interval was selected at random, and the figure obtained was the random start (RS) that corresponded to the list of villages in column A. This random start marked the first village selected in Mucwini Sub County for the study. The remaining 14 villages in Mucwini Sub County were then selected as follows:

$\mathrm{RS}+\mathrm{S} 1, \mathrm{RS}+2 \mathrm{RS} 1, \mathrm{RS}+3 \mathrm{RS} 1, \mathrm{RS}+4 \mathrm{~S} 1, \mathrm{RS}+5 \mathrm{~S} 1, \mathrm{RS}+6 \mathrm{~S} 1, \mathrm{RS}+7 \mathrm{~S} 1, \mathrm{RS}+8 \mathrm{~S} 1, \mathrm{RS}+9 \mathrm{~S} 1, \mathrm{RS}+10 \mathrm{~S} 1$, $R S+11 S 1, R S+12 S 1, R S+13 S 1, R S+14 S 1$.

The same procedure was repeated in Kitgum Matidi Sub County until 15 villages were selected for the study. In Kitgum Matidi Sub County, the following villages were selected: Pakumu A, Bobbi A, PagwaAwere, Parwech-lujul, Pakumu-Kamdini, Nazareth, Dognam, Jerusalem, Aputubere, Mulago A, Olam-Bira, Okwongobone, Putuke west, Gore, and Lapana A. In Mucwini Sub County, the following villages were selected: Biwang west, yepa ward, obiya west, Payum A ward, Ayomolola B, Pakuba west, oruma, Icengotyena ward, Pudo central, Lagot, Lacenotonya west, Pubech East, Panyok, Lotukubor and Omuna. 


\section{Selection of households for the study}

In each village, 16 households were selected for the study, and they were identified in the following ways. The village health team members helped the research team identify the most central location in each village, and in most cases, boreholes were the most centrally located structure. Therefore, a borehole was used to identify the central location where the study was conducted for each village. The direction taken in selecting the first household for the study was determined by spinning an empty soda bottle on an even ground at the borehole, where the bottle pointed when it stopped the span was taken as the direction for the initial movement. All households in that direction were counted until the edge of that village was reached. A number between one and the total number of houses along the directional line was randomly selected, and this became the first house to be visited for the study.

After visiting the first household, the second household visited was the one nearest to the first house. The nearest household was defined as one reachable in the shortest time on foot from the household just visited. In cases where two or more households were equally near the one just visited, the one on the immediate right was chosen as a researcher who stood in the doorway of the first house while looking out.

At the household level, we asked whether the head of household was present. If the response was affirmative, she/he was requested to take part in the study after explaining to him/her the details of the study and obtaining informed consent, which was documented. This procedure was repeated until 16 participants (household heads) from each village were selected in the two sub counties.

\section{Study Variables}

\section{Dependent variable}

The differences in the community prevalence of hepatitis $E$ in the two sub counties of Mucwini and Kitgum Matidi in Kitgum district were the outcome variables.

\section{Independent variables}

The independent variables were personal factors such as communal hand washings, water storage, household hygiene, animal rearing and excreta disposal, age, gender, level of education of the head of household, occupation, marital status, religion, and sources of income; the community factors included knowledge, attitude and practices on Hepatitis $E$, health seeking behaviors and sanitation management. The health system factors were accessibility and availability of health services, including health personnel, appropriate management of cases and health education.

\section{Data collection methods}

We collected quantitative data using a face-to-face interview using a semistructured questionnaire designed by the research team. 


\section{Data quality control}

To collect quality data in this study, research assistants were trained, familiar with the health care delivery systems, had prior experience in data collection and were identified by the Kitgum District Health Office. Research assistants were trained for two days on interviewing skills, correct recording of responses and correct method of identifying participants. The training emphasized strict adherence to procedures laid down in the proposal. Additionally, all data collection tools were translated to Acholi, the language spoken by most participants.

The instrument was pretested among community members who were not part of the main study. The result of the pretest was used to determine the reliability and consistency of the instrument. The finding from the pretest was used to determine the internal validity of questions in the questionnaire, which was found at Cronbach's $a=0.85 .{ }^{22}$ This semi-structured questionnaire (Additional file 1 ) was administered by research assistants who were trained and regularly supervised by the principal investigator. In addition, the principal investigator monitored data collection exercises by moving in the two sub counties and conducted on-spot checks to determine the progress of each research assistant until all the data collection was completed.

\section{Field data management}

Completed questionnaires were checked at the end of each day for completeness, coded and entered in Epi-info version 3.5.1. The data were cleaned and stored ready for the final analysis.

\section{Data Analysis}

The quantitative data in Epi version 3.5.1 were exported to STATA version 10.0, where the analysis was completed. In univariate analysis, frequencies and percentages of heads of household were computed per sub county. Proportions in one sub county were compared to the other using chi square tests at a statistically significant test of $5 \%(p<0.05)$.

Additionally, bivariate analysis was conducted to evaluate associations between the outcome variable and each of the health system, individual and community factors. The strength of the association was determined using the chi square test, with p-values (set at $<0.05$ ) at $95 \%$ confidence intervals.

Furthermore, a multivariable logistic regression analysis was conducted to determine factors associated with the differential prevalence of hepatitis $\mathrm{E}$ in the two sub-counties. All variables in the bivariate analysis with p-values less than or equal to 0.2 were entered into the final logistic regression model to determine the independent factors associated with the differential community prevalence of hepatitis $\mathrm{E}$.

Confounding was checked by observing whether the addition of covariate(s) in the regression model caused changes in the odds ratios of the main exposure by at least $10 \%$. Confounding was further checked by assessing whether variables that were insignificant at the bivariate level of analysis became significant at the multivariable level of analysis. 
Additionally, we tested the sensitivity of the final logistic regression model by adding covariates and conducting backward and forward adjustments of the model until the odds ratios remained stable even after adding more confounding covariates. ${ }^{23}$ After this, we took values of the adjusted odds ratios (at $95 \%$ confidence intervals) in the final model as the independent factors associated with the differential prevalence of hepatitis $E$ in the two subcounties.

\section{Ethical Considerations}

This study was approved by the Makerere University School of Public Health Higher Degrees Research and Ethics Committee and the Uganda National Council of Science and Technology (UNCST). Administrative clearance was obtained from the office of the District Health Officer of Kitgum District. Each respondent gave informed consent, and the team ensured that confidentiality of information was preserved throughout and after the study. The research team ensured that no name of respondents was recorded but unique identifiers in data collection tools.

\section{Results}

\section{Socio-demographic characteristics of respondents}

Participants' age ranged from 18-84 years, with a modal age group of 29-39 years and a mean age of 39.8 years $(95 \% \mathrm{Cl}: 38.4-41.2 ; \mathrm{p}=0.582)$. More than half of respondents $(247,52 \%)$ had attained a primary level of education. There was no statistically significant difference between marital status $(p=0.062)$, religion ( $p=0.484)$, level of education $(p=0.448)$, and level of income $(p=0.548)$ among respondents in the two sub counties. There were 234 (49.26\%) respondents from Mucwini and 241 (50.74\%) from Kitgum Matidi Sub Counties (Table 1).

\section{Table 2: The prevalence of hepatitis $E$ in the two subcounties in Kitgum district}

As shown in Table 2, there was a significantly higher prevalence of hepatitis E in Mucwini Sub County $97(41.99 \%)$ than in Kitgum Matidi Sub County 63 (26.47\%), $\chi^{2}=12.56 ; p<0.001$. The risk of suffering from hepatitis E was 1.61 times higher in Mucwini than in Kitgum Matidi ( $95 \% \mathrm{Cl}: 1.39-1.87 ; \mathrm{p}<0.001$ ), and being a resident of Mucwini was positively associated with suffering from hepatitis $E$.

\section{Factors associated with the higher prevalence of Hepatitis E in Mucwini Sub County}

As shown in Table 3, the main individual factors associated significantly with differential community prevalence of Hepatitis $E$ were frequency of communal hand washing $\chi^{2}=16.13, p=0.001$; hand washing after visiting a latrine $\chi^{2}=7.16, p=0.007$; communal source of drinking water $\chi^{2}=10.26, p=0.016$; drinking water from the same storage vessel $\chi^{2}=9.7, p=0.021$ and the type of domestic animals kept at home $\chi^{2}=20.5, p<0.001$. 
Table 4 shows the main health system factors that were significantly associated with the differential prevalence of hepatitis $E$ in Mucwini Sub County. These were mainly due to how health workers handled patients in their health facilities $\chi^{2}=17.17 ; p=0.001$ and the frequency of village health meetings held on hepatitis $E \chi^{2}=12.44 ; p=0.006$.

Table 5 shows community factors associated significantly with the differential prevalence of hepatitis $\mathrm{E}$ in the two communities. Knowledge of the cause of the disease $\chi^{2}=12.37, p=0.015$ and community perception of the seriousness of the disease $\chi^{2}=37.45, p<0.001$ were the main factors.

Table 6 shows the results of hepatitis E prevention practices in the two communities of Kitgum Matidi and Mucwini Sub Counties. The most significant findings were hand washing after visiting a latrine $\chi^{2}=7.16, p=0.007$, which was more frequent among residents of Kitgum Matidi than Mucwini. In addition, homestead practices to control the spread of hepatitis $E$ were better in Kitgum Matidi than in Mucwini Sub County $\chi^{2}=32.16, p=0.000$.

Table 7 shows the results of the multivariable logistic regression analysis and the independent predictors of the differential prevalence of hepatitis $E$ in the two subcounties. These were frequency of communal hand washings ( $A \circ R=0.53,95 \% \mathrm{Cl}: 0.330-0.860 ; p=0.01$ ), hand washing after visiting a latrine $(A o R=0.23$, 95\%Cl:0.110-0.860; $p=0.003)$, patients' handling by health workers (AoR=1.91, 95\%Cl:1.400-2.610; $p<0.001)$, the number of times villagers held health meetings (AoR $=0.69,95 \% \mathrm{Cl}: 0.560-0.850 ; p=0.001)$, and knowledge on the cause of the disease (AoR=1.42, 95\%Cl:0.710-1.880; $p=0.015)$.

\section{Discussions}

\section{Differential prevalence of hepatitis $E$ in the two communities}

The most considerable finding from this study was a significantly higher prevalence of hepatitis $E$ in Mucwini Sub County compared to Kitgum Matidi Sub County of Kitgum District in Northern Uganda (Figure 1). Residents of Mucwini were nearly twice as likely to suffer from Hepatitis E compared to Kitgum Matidi (Table 1, Table 2). This meant that even within the same district of Kitgum, the risk of Hepatitis E infection among households in the two communities was different, with a greater risk among residents of Mucwini Sub County.

This current finding is consistent with previous studies conducted in two sub-counties of Madi Opei and Paloga in Lamwo District, where there were more cases of hepatitis E in Paloga than in Madi Opei. ${ }^{15,16,17}$ At the time of the investigation, Paloga, a sub-county with an estimated population of approximately 11,000 , including those who lived in the main IDP camps, interim return sites (satellite camps), and original villages, experienced a rapid increase in cases due to its proximity to Madi Opei Sub County, the first affected locality with hepatitis $\mathrm{E}$ in the region. ${ }^{15}$ Conditions in the main camps in Paloga were crowded, with poor sanitation and limited numbers of communal latrines that were located far on the 
periphery of the IDP camps. ${ }^{15}$ These factors affected the disposal of wastes among residents of Paloga IDP camps, and this may have contributed to the differential prevalence in Paloga compared to Madi Opei Sub County.

\section{Individual factors associated with the differential prevalence of hepatitis $E$}

Differential community prevalence in the current study correlated with and supported by reports from the Kitgum District Health Management Information Systems, which showed a persistently higher number of hepatitis E cases in Mucwini Sub County than in Kitgum Matidi. ${ }^{18}$ Two studies conducted in Darfur in Sudan demonstrated a difference in the risk of acquiring Hepatitis E within sociodemographic characteristics, for example, age and sex, but not in communities. ${ }^{24,25}$ This current study finding contrasts with this larger outbreak of hepatitis $E$, which occurred in a displaced population of darfur where more women were hospitalized than men. ${ }^{24}$ Our study found no differences in the demographic and socioeconomic characteristics of individuals within the two communities but demonstrated a significant difference in the community prevalence of hepatitis $E$ (Table 2). We found that hand washing after visiting the latrine and the frequency of communal hand washings were associated with the differential prevalence of hepatitis $E$ in the two subcounties. Residents of Kitgum Matidi Sub County were more likely to report hand washings after the use of latrines compared to Mucwini Sub County. Residents of Mucwini were more likely to wash hands communally during ceremonies, such as funerals, weddings, traditional marriages, and community meetings, compared to their counterparts in Kitgum Matidi. The most likely reasons for the difference in the individual and household practices were partly due to the negative attitude towards hepatitis $E$ and the low commitment in enforcement of byelaws by the local council chairpersons and village health teams in Mucwini Sub County.

\section{Community factors in the differential prevalence of hepatitis $\mathrm{E}$}

Our study established that residents of Mucwini viewed hepatitis E as an infection for those with poor environmental sanitation. The local council (LC) 1 chairpersons were reported to have had disagreements with village health teams on roles and renumeration for the work done. Dissatisfied LC chairpersons did not support programs initiated by the village health teams and therefore the low supervision of hepatitis $E$ prevention practices in the affected communities. It was also observed that residents of Mucwini Sub County were less compliant with health education messages than residents of Kitgum Matidi (Table 3). In addition, residents of Mucwini were less likely to report hand washing after visiting latrines, had more frequent communal hand washings, reared domestic animals on free range, drank water from rivers, poorly disposed of excreta, incorrectly used latrine, and poorly disposed of children's feces which were consistent with previous studies conducted in Northern Uganda ${ }^{15,17}$ which demonstrated that households with two cases of Hepatitis $E$ were more likely to have a member who attended a funeral or washed hands in common basin with others and were less likely to have washed hands after defecation. ${ }^{15}$ 
This finding, however, differed from that observed in Darfur in Sudan, where poor living conditions, poor sanitary conditions and poor food security were the identified factors that contributed to the outbreak and drivers of hepatitis $\mathrm{E}$ disease in that community. ${ }^{24,25}$ The findings in this current study highlight the importance of household hygiene practices in the spread, control, and prevention of hepatitis $E$ infection in the affected communities (Table 3).

Eyasu et al. (2010) argue that hepatitis E infection could be a result of contaminated hands and suggest the need to focus on prevention strategies such as hand washing at the individual and household levels. ${ }^{15}$ Both communities under the current study reported communal hand washing practices; however, the two communities only differed in the location and age groups that adapted those traditional practices (Table 4). In Mucwini Sub County, residents were more likely to wash hands communally during ceremonies such as funerals, weddings, and traditional marriages, whereas in Kitgum Matidi, residents reported communal hand washings at the household level mainly among children (Table 3 ). This finding highlights the role of household and personal hygiene practices as important driving factors in the spread of hepatitis E infection in the affected communities (Table 3). Our current finding matches findings in a study conducted by Christopher et al. (2010) in Lamwo District in Northern Uganda, where person-toperson transmission and contaminated water sources were factors that propagated the hepatitis $E$ epidemic in the affected communities. ${ }^{17}$ That study recommended ensuring a safe drinking water supply, adequate sanitation, and proper personal and environmental hygiene as the most effective prevention and control strategies for hepatitis $\mathrm{E}$ in those communities. Additionally, two studies documented persons-to-person transmission of hepatitis $\mathrm{E}$, suggesting that strategies for prevention and control should be focused on reducing transmission through the person-to-person route. ${ }^{25,26}$

\section{Health system factors in the differential prevalence of hepatitis $\mathrm{E}$}

Our study found that professional handling of patients by health workers and frequency of village health meetings held on Hepatitis E were significantly associated with differential community prevalence of Hepatitis E in the two Sub Counties of Mucwini and Kitgum Matidi (Table 4). Residents of Kitgum Matidi were more likely to report having held many community meetings weekly and biweekly compared to Mucwini, who only held more village health meetings monthly (Table 4). Village meetings were avenues for health education on Hepatitis $E$, where information on the disease was communicated, particularly on behaviors, practices of individuals and communities as these affect their health. More frequent community health meetings were held in Kitgum Matidi Sub County to understand and educate the community on the outcomes of unhealthy behaviors and its consequences on the health of individuals, families, and communities compared to their counterparts in Mucwini (Table 4). The use of health education related to personal and household hygiene measures as conventional control efforts at the community level has also been recommended by many studies. ${ }^{17-26}$

\section{Knowledge and awareness of the causative organism of hepatitis E disease}


We found that awareness of the causal organism of hepatitis $E$ disease equipped residents with knowledge on the prevention of disease occurrence since they knew how it was transmitted. Residents of Kitgum Matidi Sub County demonstrated a higher degree of awareness regarding the etiological organism that causes the disease compared to their counterparts in Mucwini Sub County (Table 5). The adequate information they acquired could have resulted from the frequent awareness and sensitization campaigns conducted by health officials from Kitgum District and the Ugandan Ministry of Health, which were intended to educate the population about hepatitis $\mathrm{E}$ at the time when the incidence of the virus was very high in their communities (Table 5).

\section{Hepatitis E prevention practices in the two communities}

Hepatitis E prevention practices were better performed in Kitgum Matidi Sub County compared to their counterparts in Mucwini, as seen by correct hand washing after latrine use and drinking clean water, which were only $12.56 \%$ and $8.07 \%$ in Mucwini, respectively (Table 6). This difference in hepatitis $E$ prevention practices in the two subcounties may explain the differential prevalence of hepatitis $E$ in the two communities. This finding has implications for the long-term and sustainable plan for the two communities on how Kitgum District Health Office could use the information to ensure that the epidemic does not occur again, and in case it occurred, the community would be ready to handle appropriately.

\section{Independent predictors of differential hepatitis E prevalence in the two communities}

Regarding the independent determinants of hepatitis E prevalence, factors associated with differential prevalence were the frequency of communal hand washings and hand washing after the use of latrines, handling of patients by health workers, the number of times villages held health meetings and awareness of the cause of the disease (Table 7). These authors argue that continuous health education, coordinated health responses by all stakeholders, investment in clean water sources, restriction of domestic animal movement, improved sanitation and strict adherence to local health byelaws would ultimately help the community overcome the epidemic. This was similarly observed in Christopher et al. and Eyasu et al. ${ }^{15,17}$

In addition, residents of Kitgum Matidi Sub County practiced better hepatitis E prevention practices, such as correct latrine use, eating hot food, avoiding eating raw food that was not properly washed, washing hands after latrine use, avoiding communal hand washings, and drinking clean and safe water. These were, however, different from residents of Mucwini, who mainly mentioned environmental cleanliness as the major practice to control Hepatitis $\mathrm{E}$ in their community.

These authors suggest that despite these differences, the Ugandan Ministry of Health and Kitgum District health department should continue to sensitize, mobilize, and engage the two communities, especially Mucwini residents, to adopt a more progressive approach to hepatitis E control measures, including instituting byelaws to enforce the implementation of infection prevention and control of hepatitis $E$ in their community.

\section{The limitations of this study}


We used self-reported information to affirm the cases of hepatitis $E$ in the two subcounties because we did not conduct serological tests to confirm the hepatitis E virus. We used participants' medical reports from previous tests conducted by a team of infectious disease experts from the Ugandan Ministry of Health. This may have in some way led to reporting bias, which we acknowledge. We did not conduct laboratory tests at the time of the research but relied on medical reports from previous surveys conducted in the same population.

In addition, we conducted our study more than 3 years after the first case of hepatitis $E$ was confirmed in the study population and knowing that IgA and IgG only appear in the acute phase of the disease and that IgG that usually persists in a population may not be indicative of a recent infection of hepatitis $E$ in the area. Therefore, the lack of laboratory tests for the study population may not limit the findings of our study, and we think the data generated were useful for a study in remote and low-resource settings.

In conclusion, individual, community and household hygiene practices, especially hand washing after latrine use, communal hand washing, community health responses and community awareness, were the main driving factors associated with the differential prevalence of hepatitis $E$ in the two communities. There is a need to improve the frequency and depth of sensitization meetings for the affected communities, and this should be conducted jointly with village health team members and local council chairpersons at the village and parish levels to improve the level of community awareness on hepatitis $E$ to minimize the spread of the virus.

\section{Abbreviations}

HEV

Hepatitis E virus

RNA

Ribonucleic Acid

IDP

Internally displaced peoples camps

HMIS

Health Management Information System

$\mathrm{HC}$

Health Centre

PNP

Private and not-for-profit

WHO

World Health Organization

EPI

Expanded Program for Immunization

PPS

Probability Proportional to size 
UNCST

Uganda National Council of Science and Technology

LC

Local Council

$\lg \mathrm{A}$

Immunoglobulin A

$\lg G$

Immunoglobulin G

\section{Declarations}

Ethical approval and consent to participate: This study was approved by the Makerere University School of Public Health Higher Degrees Research and Ethics Committee, Uganda National Council of Science and Technology (UNCST). Administrative clearance was obtained from the office of the District Health Officer of Kitgum District. We obtained informed consent from individual participants in this study.

Consent for publication: All participants consented to the publication of this information.

Availability of data and materials: The minimal data that support this manuscript are available and can be accessed upon reasonable request to the corresponding author.

Competing Interests: All authors declare no conflicts of interest.

Funding: We are sincerely grateful to PeriPeri for financial support for this study. We, however, wish to state that Periperi did not participate in the design, collection, analysis, interpretation of data or in writing the manuscript for this study.

Authors' contributions: JA, CGO, JK participated in designing the study, JA and DLK were responsible for field work supervision, JA, CGO, DLK and ENI were responsible for data analysis, interpretation, writing and revising the manuscript.

Acknowledgements: We thank the District Health Officer of Kitgum Dr. Alex Olwedo and the district health team for providing us with the field logistic support throughout the data collection period. We thank the research assistants Mr. Ocitti Denis, Ms. Aciro Sarah, Ms. Anena Sunday, and Mr. Richard Amone for the dedication exhibited during data collection.

Authors' Information: JA is a public health specialist and works as a Technical Director for USAID, University Research Council (URC)-Rhites Acholi, Gulu City, Uganda; JK is a lecturer at Makerere University School of Public Health, Kampala, Uganda; CGO is a professor of Public health at the Makerere University, School of Public Health, Department of Community and Behavioral Sciences, Kampala, Uganda; ENI is a Technical Director and Adjunct Professor at the ICAP in University of Columbia, Free town, Sierra Leone and DLK is Professor at the Gulu University, Faculty of Medicine, Department of Surgery, Gulu City, 
Uganda and a Takemi Fellow of the Harvard University, T.H. Chan School of Public Health and Populations.

\section{References}

1. World Health organization. Hepatitis E. Document WHO/CDSCSR/EDC/2002.12. Geneva, Switzerland. World Health Organization; 2001. Department of communicable disease surveillance and response, 2001.

2. Balayan MS. Epidemiology of Hepatitis E virus infection. Journal of Viral Hepatitis.1997;4:155-6.

3. San-Shwe, Myint-Myint-Soe. Epidemiological criteria and indication of non-A, non-B hepatitis in a community. Lancet.1985;2:828

4. Skidmore SJ. Overview in the spread of Hepatitis E. Current Infectious Disease report. 2002;4(2):118-123

5. Skidmore SJ. Factors in the spread of Hepatitis E. Lancet. 1999; 354:1049-50

6. Centers for Disease Control and Prevention (CDC). Enterically transmitted non-A, non-B hepatitis in East Africa. MWMR morbidity Mortality Weekly report. 1987;36:241-4

7. SciTechDaily. Hepatitis E Vaccine debuts to Chinese Biotech Partnership. ScienceNews, Diseases, Epidemiology, Vaccine, Virology. 2012.

8. Vishwanathan R. Infectious Hepatitis E in New Delhi, 1955-1956 a critical study: Epidemiology. Indian Journal of Medical Research. 1957;45(suppl. 1):1-29

9. Lewis HC, Boissons S, ljazs et al., Hepatitis E in England and Wales. Emerging Infectious Diseases. 2008; $14: 165-7$

10. Amon JJ, Drobenuic J, Bower WA et al., Locally acquired Hepatitis E virus infection, El Paso, Texas. Journal of Medical Viriology. 2006; 78:741-6

11. Teo CG. Hepatitis E. In: Centers for Disease control and Prevention. Health information for international travel. Atlanta: USA Department of health and Human Services, Public Health services. 2009:335-8

12. Gouvea V, Snellings N, Popek MJ, Longenr CF, Innis BL. Hepatitis E virus: Complete genome sequencing and phylogenetic analysis of Nepal isolates. Viral Research. 1988; 57:21-6

13. Shrestha SM, Tsuda F, Nishizawa T, Gotanda Y, Takda N, Okamoto H. Molecular investigations of Hepatitis $\mathrm{E}$ virus infections in patients with acute hepatitis in Kathmandu. Nepal Journal of Medical virology. 2003; 69:207-14

14. Teshale EH, Howard CM, Grytdal SP, et al., Hepatitis Epidemic, Uganda. Emerging Infectious Diseases. 2010; 16:126-9

15. Eyasu H, Christopher MA, Scott P, Grytdal SP, et al., Evidence of person-to-person transmission of Hepatitis E during a large outbreak in northern Uganda. Clinical infectious Disease; 2010;50(7):1006-10 
16. WHO. Update on the Hepatitis E epidemic in Kitgum district. 2008. Kitgum district. Hepatitis E surveillance report. .

17. Christopher MH, Thomas H, Vincent R, Hill SP, et al., Novel risk factors associated with Hepatitis $\mathrm{E}$ virus infection in large outbreak in northern Uganda, results from a case control study and environmental analysis. American Journal of Tropical Medicine and Hygiene. 2010; 83(5):1170-3

18. WHO. Kitgum District Surveillance report on Hepatitis E; Health Management Information System report of Kitgum district. 2010.

19. Campbell MJ, Julious SA, Altman DG. <background-color:\#CCCCFF;uvertical-align:super;>Estimating sample sizes for binary, ordered categorical, and continuous outcomes in two group comparisons</background-color:\#CCCCFF;uvertical-align:super;>. Bmj.1995;311(7013):1145-1148

20. Bland JM. Sample size in guidelines trails. Fam pract. 2000;17 suppl. 1.S17-20.

21. World Health Organization (WHO). Training for mid-level Managers: The EPI Coverage Survey. WHO document WHO/EPI/MLM/91.10 Geneva. World Health Organization. 1991. Available: http.//whqlibdoc.who.int/hq/1991/WHOEPIMLM91.10.pdf. accessed on March 2012.

22. Lee J Cronbach and Paul E Meehl. Construct Validity in Psychological Tests. First published in Psychological Bulletin.1955;52:281-302

23. Saltelli A, Ratto M, Andres T, Lampalongo F, Cariboni J, Gatelli D, Saisana M, Tarantola S. Global sensitivity analysis; the Primer. John Wiley and Sons Ltd, 2008. Library of congress cataloguing-inpublication data.

24. Boccia D, Guthmann JP, Klovstad H, Hamid N, et al., High mortality associated with an outbreak of Hepatitis E among displaced persons in Darfur, Sudan. Clinical infectious Diseases. 2006; 42:167984

25. Guthmann JP, Hilde K, Boccia D, et al., A large outbreak of Hepatitis E among a displaced population in Darfur, Sudan 2004: The role of water treatment methods. Clinical Infectious Diseases. 2006;42:1685-91

26. Eyasu H Teshale, Dale J Hu, Scott D Holmberg, James M Hughes, and Mary E Wilson. The two faces of Hepatitis E virus. Clinical Infectious Disease. 2011;51:328-34

\section{Tables}

Others $^{(a)}$ are single, separated and divorced; others ${ }^{(b)}$ are Muslims, Pentecostals, and SDA; others ${ }^{(c)}$ are formal employment.

Table 1 shows that the sociodemographic characteristics of respondents in the two sub counties were comparable, and there was no statistically significant difference. 
Table 1: Socio-demographic characteristics of respondents in Kitgum Matidi and Mucwini Sub counties

$\begin{array}{lllll}\text { Variables } & \text { Mucwini }(n=234) & \text { Kitgum Matidi }(n=241) & \chi^{2} & \text { p-value } \\ & \text { Frequency }(\%) & \text { Frequency }(\%) & \end{array}$

Gender

\begin{tabular}{lllll} 
Male & $98(41.88)$ & $94(39.00)$ & 0.517 & 0.42 \\
Female & $136(58.12)$ & $147(61.00)$ & & \\
\hline
\end{tabular}

\section{Age-groups}

\begin{tabular}{lllll}
\hline $18-28$ & $62(26.50)$ & $78(32.37)$ & 1.952 & 0.582 \\
\hline $29-39$ & $65(27.78)$ & $68(28.22)$ & & \\
\hline $40-50$ & $50(21.37)$ & $51(21.16)$ & & \\
$>50$ & $55(23.50)$ & $52(21.58)$ & & \\
\hline Marital status & & & & 0.9805 \\
\hline Married & $162(69.23)$ & $194(80.50)$ & & \\
\hline Widowed & $32(13.68)$ & $20(8.30)$ & & \\
\hline Others $^{(a)}$ & $38(16.24)$ & $27(11.20)$ & & \\
\hline
\end{tabular}

Religion

Catholic

152(64.96)

162(67.22)

4.467

0.484

Protestant

77(32.91)

68(28.22)

Others $^{(b)}$

3(1.28)

8(3.32)

\section{Level of Education}

\begin{tabular}{|c|c|c|c|c|}
\hline None & $61(26.07)$ & $70(29.05)$ & 2.6534 & 0.448 \\
\hline Primary & $126(53.85)$ & $121(50.21)$ & & \\
\hline Secondary & $36(15.38)$ & $35(14.52)$ & & \\
\hline Post-secondary & $8(3.42)$ & $15(6.22)$ & & \\
\hline \multicolumn{5}{|c|}{ Sources of Income } \\
\hline Farming & $180(76.92)$ & $175(72.61)$ & 1.2026 & 0.548 \\
\hline Business & $36(15.38)$ & $38(15.77)$ & & \\
\hline Others ${ }^{(c)}$ & $9(3.85)$ & $14(5.81)$ & & \\
\hline
\end{tabular}


Table 2: Two by two table showing proportion of the household that reported to have had a member of the family having had Hepatitis E by Sub County

\section{Suffered from Hepatitis E}

\begin{tabular}{llll}
\hline Sub County & No & Yes & Total \\
\hline Kitgum Matidi & $175(73.53 \%)$ & $63(26.47 \%)$ & $238(100 \%)$ \\
\hline Mucwini & $134(58.01 \%)$ & $97(41.99 \%)$ & $231(100 \%)$ \\
\hline Total & $309(65.88 \%$ & $160(34.12 \%)$ & $469(100 \%)$
\end{tabular}

Table 2 shows a two-by-two table and the reported prevalence of hepatitis $\mathrm{E}$, which was higher in Mucwini Sub County 97 (41.99\%) than in Kitgum Matidi 63 (26.47\%) at 95\% confidence interval: $\chi^{2}=1.61,95 \% \mathrm{Cl}$ : $1.39-1.87 ; p<0.001$ 
Table 3: Bivariate analysis of individual factors associated with differential community prevalence of Hepatitis $\mathrm{E}$ in Kitgum district

Variables of Interest
Kitgum Matidi

$(n=241)$

Frequency (\%)
Mucwini ( $n=234)$

Frequency (\%) $x^{2} \quad p$-value

$2.65 \quad 0.448$

61(26.07)

126(53.85)

36(15.38)

$8(3.42)$

15(6.22)

149(61.83)

132(56.41)

1.59

0.207

Communal hand washing

Frequency of Communal hand washing

\begin{tabular}{lllll}
\hline Everyday & $119(49.38)$ & $103(44.02)$ & 16.13 & $\mathbf{0 . 0 0 1 *}$ \\
\hline During Ceremonies & $11(4.56)$ & $31(13.25)$ & & \\
\hline Water scarcity & $3(1.24)$ & $4(1.71)$ & & \\
\hline Others & $3(1.24)$ & $3(1.28)$ & & \\
\hline $\begin{array}{l}\text { Hand washing before and after } \\
\text { eating food }\end{array}$ & $235(97.51)$ & $224(95.73)$ & 1.12 & 0.288 \\
\hline $\begin{array}{l}\text { Hand washing after visiting the } \\
\text { toilet }\end{array}$ & $229(95.02)$ & $206(88.03)$ & 7.16 & $\mathbf{0 . 0 0 7 *}$ \\
\hline $\begin{array}{l}\text { Hand washing with soap after } \\
\text { visiting the toilet }\end{array}$ & $106(43.98)$ & $118(50.43)$ & 2.13 & 0.144 \\
\hline
\end{tabular}

\section{Excreta disposal}

Dig hole

71(29.46)

55(23.50)

6.598

0.086

Use the bush

33(13.69)

25(10.68)

Use latrine

135(56.02)

148(63.25)

Share

$0(0.00)$

$3(1.28)$

\section{Sources of drinking water}

Bore hole

River

234(97.10)

$1(0.41)$
220(94.02)

10.29

$0.016 *$ 
Protected Spring

Un protected spring

Water storage for drinking

\begin{tabular}{lllll} 
Saucepan & $3(1.24)$ & $6(2.56)$ & 9.734 & $\mathbf{0 . 0 2 1 ^ { * }}$ \\
\hline Wide mouth pot & $52(21.58)$ & $30(12.82)$ & & \\
\hline Jerry can & $177(73.44)$ & $193(82.48)$ & & \\
\hline Others & $8(3.32)$ & $3(1.28)$ & 20.5 & $\mathbf{0 . 0 0 0 0 *}$ \\
\hline Domestic animal kept at home & & & & \\
\hline Donkeys & $4(1.66)$ & $2(0.85)$ & & \\
\hline Goats & $151(62.66)$ & $127(54.27)$ & & \\
\hline Pigs & $18(7.47)$ & $34(14.53)$ & & \\
\hline Others & $34(14.11)$ & $50(21.37)$ & $11(4.70)$ & \\
\hline None & $32(13.28)$ & & & \\
\hline
\end{tabular}

*Significant finding at $p<0.05$

Table 3 shows the statistically significant individual variables at $95 \%$ confidence intervals: rearing of domestic animals $\chi^{2}=20.5, p<0.0001$; community water storage for drinking $\chi^{2}=9.734$, $p=0.021$; sources of drinking water $\chi^{2}=10.29 ; p=0.016$, hand washing after visiting a latrine $\chi^{2}=7.16$, $p=0.007$ and frequency of communal hand washings $\chi^{2}=16.13, p=0.001$. 
Table 4: Health systems factors associated with differential community prevalence of Hepatitis $E$ in Kitgum District
Variables of Interest
Kitgum Matidi ( $\mathrm{n}=241)$
Mucwini ( $\mathrm{n}=234)$
$\chi^{2}$
p-value
Frequency (\%)
Frequency (\%)

Distance from a health unit

$\begin{array}{lllll}<1 \mathrm{~km} & 152(63.07) & 132(56.41) & 1.8806 & 0.391 \\ 1-5 \mathrm{~km} & 62(25.70) & 70(29.91) & & \\ >5 \mathrm{~km} & 27(11.20) & 30(12.82) & & \end{array}$

Hepatitis E unit set up

\begin{tabular}{lllll} 
None & $172(71.37)$ & $165(70.51)$ & 1.0491 & 0.592 \\
\hline 1 unit & $66(27.39)$ & $67(28.63)$ & & \\
$>1$ unit & $1(0.041)$ & $0(0.00)$ &
\end{tabular}

The kind of reception by health workers at the treatment centers

\begin{tabular}{lllll} 
Very well & $1(0.041)$ & $1(0.043)$ & 17.17 & $\mathbf{0 . 0 0 1 *}$ \\
\hline well & $53(21.99)$ & $85(36.32)$ & & \\
\hline Fair & $72(29.88)$ & $74(31.62)$ & \\
\hline Poor & $115(47.72)$ & $72(30.77)$ &
\end{tabular}

Frequency of village health meetings held on Hepatitis $E$

\begin{tabular}{lllll} 
None & $144(59.75)$ & $108(46.15)$ & 12.44 & $\mathbf{0 . 0 0 6 *}$ \\
\hline Every week & $22(9.13)$ & $19(8.12)$ & \\
\hline Every two weeks & $32(13.28)$ & $31(13.25)$ & \\
\hline Once every month & $43(17.84)$ & $72(30.77)$ &
\end{tabular}

*Significant finding $p<0.05$

Table 4 shows that the significant health system factors were the frequency of village health meetings held $\chi^{2}=12.44, p=0.006$ and the handling of patients by health workers $\chi^{2}=17.17, p=0.001$. 
Table 5: Health systems factors associated with differential community prevalence of Hepatitis $E$ in Kitgum District

Variables of Interest

\section{Level of awareness}

Know the disease

Do not know

Knowledge on causative organism

Germ (Bacteria)

Houseflies

Dirty environment

Eating dirty food

Do not know

Level of sanitation

Latrine at home

Health seeking behavior

Treatment received from

Health unit

Local herbs

None

Others

Perception of the seriousness of the disease

Strongly Agree

Agree

Not sure

Disagree

Strongly Disagree

*Significant finding $p<0.05$

$1(0.41)$

$5(2.05)$

$3(1.24)$
$1(0.41)$
$5(2.05)$
isease

$6(2.49)$

72(29.88)

$5(2.07)$
Kitgum Matidi $(n=241)$

Frequency (\%)

Mucwini $(n=234) \quad \chi^{2} \quad p$-value

Frequency (\%)

130(53.94)

130(55.56)

$3.158 \quad 0.368$

109(45.22)

100(42.74)

24(9.96)

41(17.87)

12.37

$0.015^{*}$

42(17.43)

31(13.36)

125(51.87)

131(56.47)

47(19.50)

29(12.50)

2(0.830)

$0(0.00)$

201(83.40)

187(80.60)

1.209

0.271

0.299

7(2.99)

1(0.04)

2(0.09)

47(20.09)

37.45

0.000 *

107(45.73)

18(7.69)

55(23.50)

$4(1.71)$

\begin{tabular}{ll}
$132(54.77)$ & $107(45.73)$ \\
\hline $24(9.96)$ & $18(7.69)$ \\
\hline $72(29.88)$ & $55(23.50)$ \\
$5(2.07)$ & $4(1.71)$
\end{tabular}


In Table 5, perception of the community on the seriousness of the disease $\chi^{2}=37.45, p<0.0001$ and knowledge on the causative organism $\chi^{2}=12.37, p=0.015$ were statistically significant factors in the two subcounties.

Table 6: Hepatitis E prevention practices in the communities of Kitgum Matidi and Mucwini Sub Counties in Kitgum District

\begin{tabular}{|c|c|c|c|c|}
\hline \multirow[t]{2}{*}{ Hepatitis E prevention practices } & $\begin{array}{l}\text { Kitgum Matidi } \\
(n=241)\end{array}$ & $\begin{array}{l}\text { Mucwini } \\
(n=234)\end{array}$ & $x^{2}$ & $\begin{array}{l}\text { p- } \\
\text { value }\end{array}$ \\
\hline & Frequency (\%) & \multicolumn{3}{|l|}{ Frequency (\%) } \\
\hline Ownership of latrine at home & $201(83.40)$ & 187(79.91) & 1.20 & 0.27 \\
\hline $\begin{array}{l}\text { Hand washing before and after } \\
\text { eating }\end{array}$ & 235(97.51) & 224(95.73) & 1.13 & 0.28 \\
\hline Hand washing after visiting latrine & $229(95.02)$ & $206(88.03)$ & 7.16 & $0.007 *$ \\
\hline \multicolumn{5}{|l|}{ Disposal of children's faeces } \\
\hline Latrine & $169(70.12)$ & $145(61.97)$ & 5.79 & 0.122 \\
\hline Dig hole & $64(26.56)$ & $76(32.48)$ & & \\
\hline Use bush & $5(2.07)$ & $0(0.00)$ & & \\
\hline \multicolumn{5}{|c|}{ Practices of Hepatitis E control in homestead } \\
\hline Correct latrine use & $50(20.75)$ & 28(11.97) & 32.16 & $0.000 *$ \\
\hline Keep the environment clean & $89(36.93)$ & $139(59.40)$ & & \\
\hline Eating hot food & $35(14.52)$ & $22(9.40)$ & & \\
\hline Drinking clean/safe water & $15(6.22)$ & 18(7.69) & & \\
\hline Wash hands after visiting latrine & $31(12.86)$ & $10(4.27)$ & & \\
\hline None & $3(1.24)$ & $6(1.71)$ & & \\
\hline
\end{tabular}

*Significant finding, $p<0.05$

In Table 6, homestead practices on the control of hepatitis $E$, such as correct latrine use $\chi^{2}=32.16$, $p<0.0001$ and hand washing after visiting the latrine $\chi^{2}=7.16, p=0.007$, were statistically significant factors in the two communities. 
Table 7: Multivariable logistic regression analysis with variables significant at the bivariate analysis

\begin{tabular}{|c|c|c|c|c|c|}
\hline Variable & $\begin{array}{l}\text { B- } \\
\text { coefficient }\end{array}$ & $\begin{array}{l}\text { Standard } \\
\text { error }\end{array}$ & $\begin{array}{l}\text { Adjusted } \\
\text { Odds ratios }\end{array}$ & $\begin{array}{l}\mathrm{p}- \\
\text { value }\end{array}$ & $\begin{array}{l}95 \% \\
\mathrm{Cl}\end{array}$ \\
\hline $\begin{array}{l}\text { Frequency of Communal hand } \\
\text { washings }\end{array}$ & -2.57 & 0.13 & 0.53 & $0.01^{*}$ & $\begin{array}{l}0.330- \\
0.860\end{array}$ \\
\hline hand washing after visiting latrine & -2.94 & 0.12 & 0.23 & $0.003^{*}$ & $\begin{array}{l}0.110- \\
0.646\end{array}$ \\
\hline Sources of drinking water & 0.63 & 0.45 & 1.26 & 0.53 & $\begin{array}{l}0.610- \\
2.540\end{array}$ \\
\hline Drinking water from a storage vessel & -1.54 & 0.17 & 0.67 & 0.124 & $\begin{array}{l}0.410- \\
1.110\end{array}$ \\
\hline Handling by health workers & 4.09 & 0.3 & 1.91 & $0.000 *$ & $\begin{array}{l}1.400- \\
2.610\end{array}$ \\
\hline $\begin{array}{l}\text { Frequency of village health meetings } \\
\text { held }\end{array}$ & -3.47 & 0.074 & 0.69 & $0.001 *$ & $\begin{array}{l}0.560- \\
0.850\end{array}$ \\
\hline $\begin{array}{l}\text { Perception on the seriousness of the } \\
\text { disease }\end{array}$ & 0.11 & 0.18 & 1.02 & 0.912 & $\begin{array}{l}0.710- \\
1.460\end{array}$ \\
\hline $\begin{array}{l}\text { Knowledge on the causative } \\
\text { organism of Hepatitis } E\end{array}$ & 2.44 & 0.2 & 1.42 & $0.015^{*}$ & $\begin{array}{l}0.710- \\
1.880\end{array}$ \\
\hline
\end{tabular}

*Significant finding, $\mathrm{p}<0.05$

In table 7, the independent predictors associated with differential prevalence of Hepatitis $E$ in Mucwini Sub County were frequency of communal hand washings $A o R=0.530,95 \% \mathrm{Cl}: 0.330-0.860 ; p=0.01$; hand washing after visiting a latrine $A o R=0,230,95 \% \mathrm{Cl}$ : $0.110-0.646 ; p=0.003$; handling of patients by health workers $A o R=1.910,95 \% \mathrm{Cl}: 1.400-12.610 ; p=0.000$; frequency of village health meetings held $A o R=0.690$, 95\%Cl: $0.560-0.850 ; p=0.001$; and knowledge on the causative organism to Hepatitis $E \mathrm{AoR}=1.42,95 \% \mathrm{Cl}$ : $0.710-0.880 ; p=0.015$

\section{Figures}




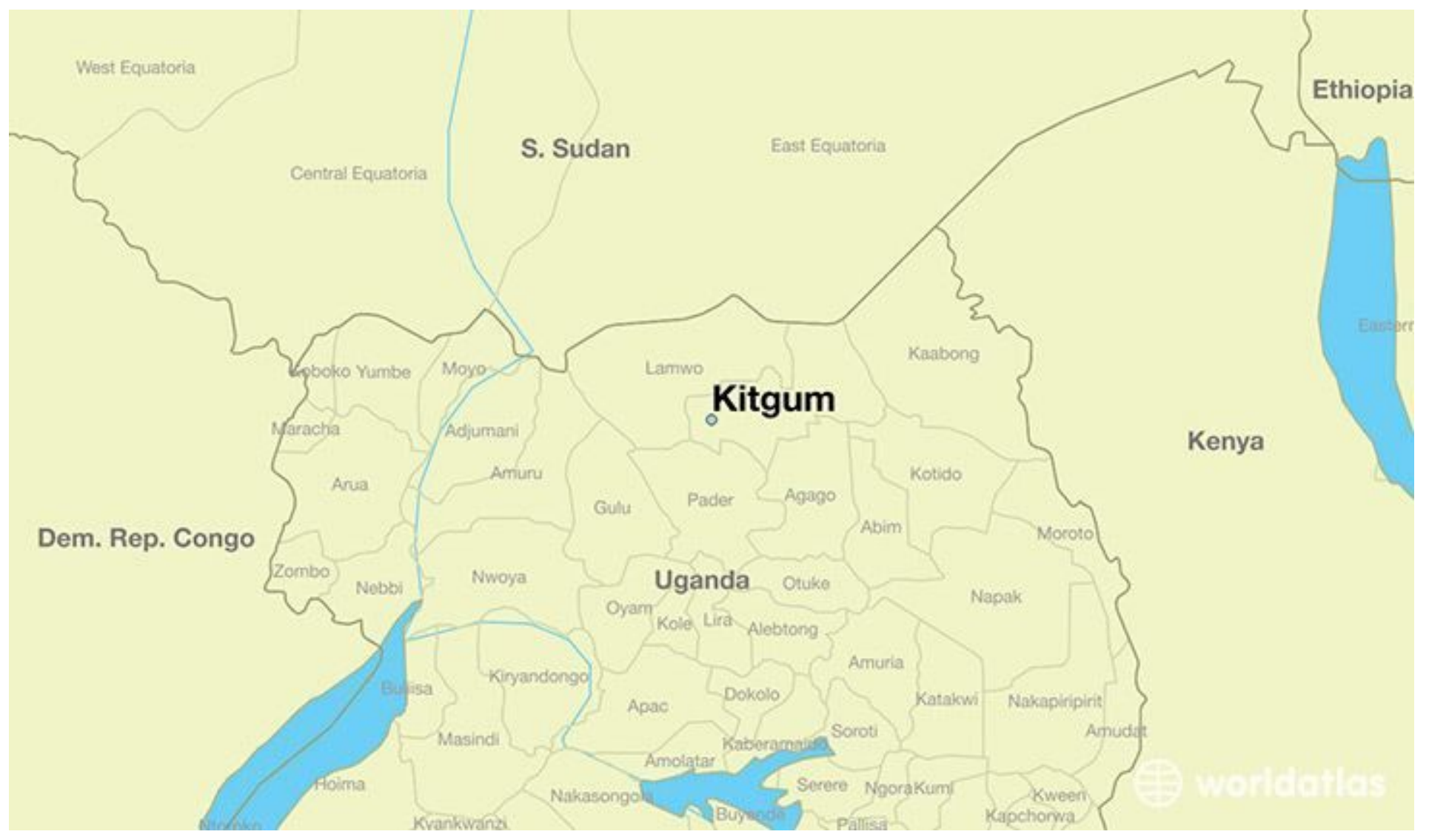

\section{Figure 1}

The Map of Kitgum district in Northern Uganda. Figure 1 shows a map of the Kitgum district in northern Uganda. This was

the site for study in the Sub Counties of Mucwini and Kitgum Matidi.

\section{Supplementary Files}

This is a list of supplementary files associated with this preprint. Click to download.

- SupplementaryAppendixA12.docx 